\title{
ROTATIONAL SPECTRA OF SMALL PAHS: ACENAPHTHENE, ACENAPHTHYLENE, AZULENE, AND FLUORENE
}

\author{
S. Thorwirth, ${ }^{1,2,3}$ P. Theulé,,${ }^{2,3,4}$ C. A. Gottlieb, ${ }^{2,3}$ M. C. McCarthy, ${ }^{2,3}$ and P. Thaddeus ${ }^{2,3}$ \\ Received 2007 January 5; accepted 2007 March 7
}

\begin{abstract}
Pure rotational spectra of four small polycyclic aromatic hydrocarbons have been observed by Fourier transform microwave spectroscopy of a molecular beam in the frequency range from 7 to $37 \mathrm{GHz}$. Initial searches for acenaphthene $\left(\mathrm{C}_{12} \mathrm{H}_{10}\right)$, acenaphthylene $\left(\mathrm{C}_{12} \mathrm{H}_{8}\right)$, and fluorene $\left(\mathrm{C}_{13} \mathrm{H}_{10}\right)$ were guided by quantum chemical calculations performed at the B3LYP/cc-pVTZ level of theory. All three molecules exhibit $b$-type rotational spectra and are calculated to be moderately polar, with dipole moments of $0.3-0.9 \mathrm{D}$. Close agreement (to better than $1 \%$ ) between the calculated equilibrium and experimentally derived ground-state rotational constants is achieved. Selected transitions of acenaphthene and fluorene have also been measured in the $3 \mathrm{~mm}$ region by conventional free-space absorption spectroscopy, as have transitions of the previously studied azulene $\left(\mathrm{C}_{10} \mathrm{H}_{8}\right)$. The data presented here facilitate deep radio astronomical searches with large radio telescopes.
\end{abstract}

Subject headings: astrochemistry — ISM: molecules — molecular data

Online material: machine-readable tables

\section{INTRODUCTION}

The mid-infrared spectra from 3 to $20 \mu \mathrm{m}$ of many astronomical objects are dominated by emission features usually referred to as the unidentified infrared bands (UIRs). Owing to reasonable agreement between some of the UIRs and their vibrational spectra, polycyclic aromatic hydrocarbons (PAHs) are widely believed to be responsible molecular carriers (e.g., Leger \& Puget 1984; Allamandola et al. 1985). The problem with this PAH hypothesis is, however, that no individual PAH has been identified in space to date to yield conclusive evidence, despite all observational and laboratory investments (Peeters et al. 2004b; Salama 1999; Oomens et al. 2003; Tielens \& Peeters 2004). Here, highresolution microwave spectroscopy might play a crucial role in future astronomical investigations.

While PAHs are generally nonpolar or only weakly polar molecules, some have sufficiently large dipole moments for rotational spectroscopy, but only a few have been studied. The rotational spectrum of azulene $\left(\mathrm{C}_{10} \mathrm{H}_{8}\right)$, a structural isomer of nonpolar naphthalene, has been observed in the microwave range (Tobler et al. 1965; Huber et al. 2005). Very recently, Lovas et al. (2005) reported the Fourier transform microwave spectrum of corannulene $\left(\mathrm{C}_{20} \mathrm{H}_{10}\right)$, a nonplanar, bowl-shaped PAH. The combination of a fairly large dipole moment (2.1 D) and high molecular rigidity, causing all $K$-components of a given rotational transition to collapse to a single line, leads to a comparably strong rotational spectrum and makes this molecule a plausible target for astronomical searches with large radio telescopes.

In the following, a combined theoretical and experimental rotational spectroscopic investigation of selected simple PAHs $\mathrm{C}_{n} \mathrm{H}_{m}$ with $n \leq 13$ is presented (Fig. 1). The rotational spectra of acenaphthene $\left(\mathrm{C}_{12} \mathrm{H}_{10} ;\right.$ molecule 1 in Fig. 1), acenaphthylene

\footnotetext{
1 Max-Planck-Institut für Radioastronomie, Auf dem Hügel 69, 53121 Bonn, Germany; sthorwirth@mpifr-bonn.mpg.de.

2 Harvard-Smithsonian Center for Astrophysics, 60 Garden Street, Cambridge, MA 02138.

3 Division of Engineering and Applied Sciences, Harvard University, 29 Oxford Street, Cambridge, MA 02138.

${ }^{4}$ Physique des interactions ioniques et moléculaires, Université de Provence, Centre de Saint Jérôme, 13397 Marseille Cedex 20, France.
}

$\left(\mathrm{C}_{12} \mathrm{H}_{8}\right.$; molecule 2), and fluorene $\left(\mathrm{C}_{13} \mathrm{H}_{10}\right.$; molecule 3) have been obtained for the first time employing Fourier transform microwave (FTM) spectroscopy. Centimeter-wave transitions of azulene (molecule 4) were also measured, complementing the data reported previously by Huber et al. (2005 and references therein). Selected transitions of acenaphthene, fluorene, and azulene have also been observed at a wavelength of $3 \mathrm{~mm}$, employing free-space millimeter-wave absorption spectroscopy.

\section{EXPERIMENT AND QUANTUM CHEMICAL CALCULATIONS}

The FTM spectrometer used in the present investigation operates between 5 and $43 \mathrm{GHz}$ and has been described in detail elsewhere (McCarthy et al. 1997, 2000). All samples were purchased commercially (Sigma-Aldrich) and used without further purification (Acenaphthene 99\%, Acenaphthylene 75\%, Fluorene 98\%, and Azulene 99\%). Since they are all solids at room temperature, experiments were carried out using a heated nozzle recently developed for measurements of compounds of low volatility (Thorwirth et al. 2005). The nozzle was operated at temperatures from $100^{\circ} \mathrm{C}$ to $130^{\circ} \mathrm{C}$, with neon as the buffer gas.

In the millimeter-wave region, selected transitions of azulene, acenaphthene, and fluorene were measured in a free-space glass cell (1.5 m long and $7 \mathrm{~cm}$ in diameter) with a spectrometer described elsewhere (Gottlieb et al. 2003). Measurements of azulene were done at room temperature, those of acenaphthene and fluorene at room temperature and slightly above (to $50^{\circ} \mathrm{C}$ ). The effect of heating was found to be small.

Initial searches for rotational transitions were based on quantum chemical calculations of rotational constants and dipole moments performed with the standard program package GAUSSIAN03 (Frisch et al. 2003) using the density functional theory (DFT) variant B3LYP (Becke 1993) and the correlation-consistent polarized valence basis set cc-pVTZ (Dunning 1989).

\section{RESULTS}

Fitting of experimental data was done with the SPFIT program (Pickett 1991), a Watson Hamiltonian in the $A$ reduction, and either the $I^{r}$ (azulene, fluorene) or $I I^{l}$ (acenaphthene, 
<smiles>c1cc2c3c(cccc3c1)CC2</smiles>
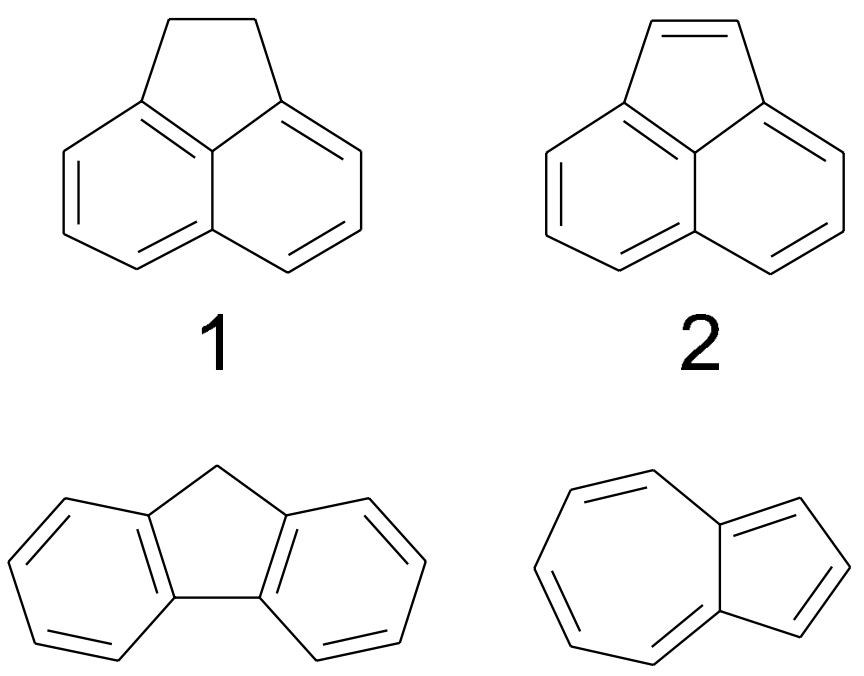

3
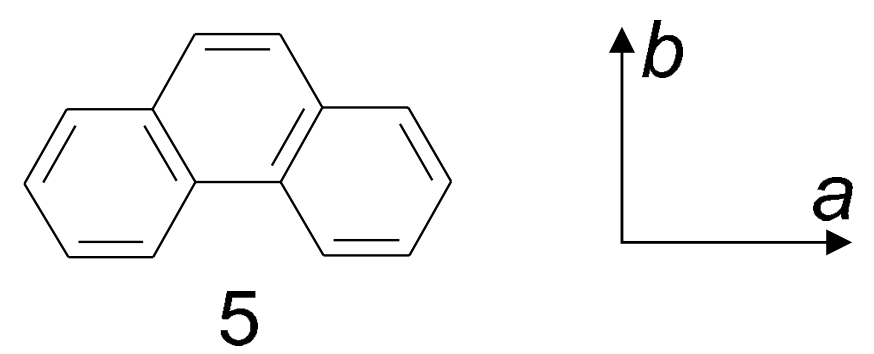

FIG. 1.-PAHs investigated here: acenaphthene ("1"), acenaphthylene ("2"), fluorene (" 3 "), azulene ("4"), and phenanthrene ("5"). All molecules are of $\mathrm{C}_{2 \mathrm{v}}$ symmetry. Except for azulene, whose dipole moment is oriented along the $a$-axis, all other molecules exhibit $b$-type rotational spectra.

acenaphthylene) reduction. The estimated experimental uncertainties $(1 \sigma)$ are $2-5 \mathrm{kHz}$ in the microwave band and $20-40 \mathrm{kHz}$ in the millimeter-wave band.

\subsection{Azulene}

Azulene (Fig. 1, molecule 4) is a somewhat prolate asymmetric top ${ }^{5}(\kappa=-0.61)$ with an $a$-type rotational spectrum. In the present study, azulene was used as a test molecule to check the feasibility of observing rotational spectra of PAHs. Transitions were found already at moderate temperatures of the heated nozzle of about $80^{\circ} \mathrm{C}$ based on predictions from the parameter set given in Huber et al. (2005). Forty one centimeter-wave lines were then newly measured at a temperature of $100^{\circ} \mathrm{C}$ between 5.8 and $23 \mathrm{GHz}$, with angular momentum quantum numbers $3 \leq J \leq 12$ and $0 \leq K_{a} \leq 6$. Based on predictions from the resulting improved set of molecular parameters, rotational transitions were also measured in the millimeter-wave band. A sample spectrum of azulene at $91.9 \mathrm{GHz}$ is shown in Figure 2. Table 1 gives 45 lines, measured from 91.4 to $94.9 \mathrm{GHz}$ (paired transitions counted once) in the millimeter-wave range, together with the new centimeter-wave data. The derived rotational constants obtained from a fit to the centimeter-wave data and from a global fit to all available data are shown in Table 2. As can be seen, the

\footnotetext{
5 Ray's parameter is a measure to quantify the symmetric character of asymmetric top molecules and is defined as $\kappa=(2 B-A-C) /(A-C)$. The limiting values of $\kappa=-1$ and +1 correspond to prolate and oblate symmetric top rotors, respectively.
}

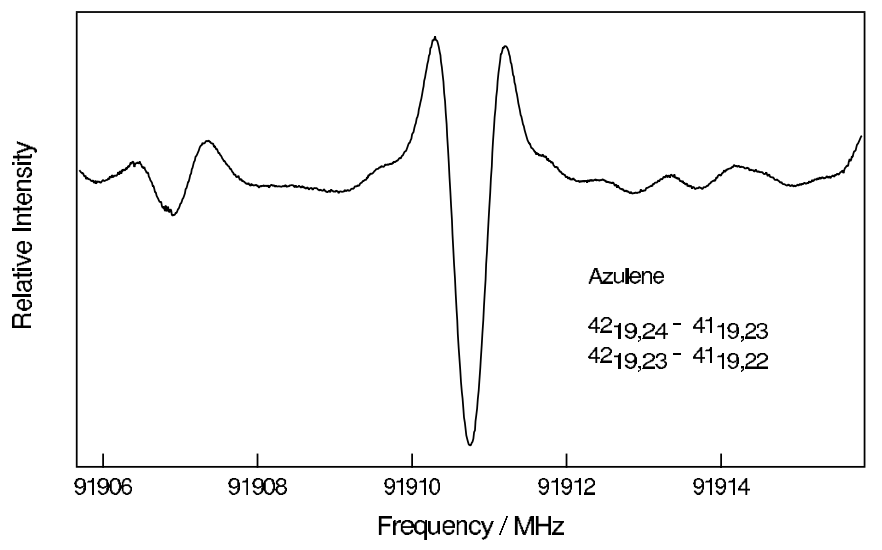

FIG. 2.- Sample spectrum of azulene at $91.9 \mathrm{GHz}$.

calculated equilibrium rotational constants are slightly larger, but well within $1 \%$ of the experimental ground-state rotational constants.

\subsection{Acenaphthene}

Acenaphthene (Fig. 1, molecule 1) is a somewhat oblate asymmetric top molecule $\left(\kappa_{\mathrm{qcc}}=+0.4\right)$ with a dipole moment of $0.90 \mathrm{D}$ along the $b$-axis. Based on the rotational constants from the B3LYP/cc-pVTZ calculation (Table 4) a search for the $6_{0,6^{-}}$ $5_{1,5} / 6_{1,6}-5_{0,5}$ doublet at $8.5 \mathrm{GHz}$ was performed at a temperature of the heated nozzle of $105^{\circ} \mathrm{C}$. This doublet was chosen because of the small but well-resolved predicted splitting of the lines ( $\sim 140 \mathrm{kHz}$ ), yielding an easily recognized signature. The doublet was found to be located about $40 \mathrm{MHz}$ lower in frequency than predicted (Fig. 3). In addition to the $J=6-5$ doublet, the initial survey scan also showed two more lines that, because of both intensity and separation, were assigned to the $3_{3,0}-2_{2,1}$ and $5_{1,4}-4_{2,3}$ transitions at 8385 and $8417 \mathrm{MHz}$, respectively.

The better rotational constants which resulted from these assignments then allowed many more transitions to be found. In all, 144 transitions were measured in the centimeter-wave from 7.0 to $33.4 \mathrm{GHz}$, at quantum numbers $2 \leq J \leq 16$ and $0 \leq$ $K_{c} \leq 16$ (Table 3), which yielded the full set of quartic centrifugal distortion constants. The corresponding fit is shown in Table 4, column (3). Based on this experimental parameter set, transitions of acenaphthene were also found in the millimeter-wave region between 92 and $96 \mathrm{GHz}$, and another 45 lines were then measured

TABLE 1

Experimental Rotational Transition Frequencies of Azulene

\begin{tabular}{rccccccrc}
\hline \hline & & & & & & $\begin{array}{c}\text { Frequency } \\
(\mathrm{MHz})\end{array}$ & \multicolumn{1}{c}{$\begin{array}{c}o-c \\
(\mathrm{MHz})\end{array}$} & $\begin{array}{c}\Delta \nu \\
(\mathrm{MHz})\end{array}$ \\
\hline 3 & 1 & 3 & 2 & 1 & 2 & 5764.5899 & -0.0007 & 0.002 \\
3 & 0 & 3 & 2 & 0 & 2 & 6139.8457 & 0.0002 & 0.002 \\
3 & 1 & 2 & 2 & 1 & 1 & 6910.6951 & 0.0003 & 0.002 \\
6 & 1 & 5 & 6 & 1 & 6 & 7588.9097 & 0.0008 & 0.002 \\
4 & 1 & 4 & 3 & 1 & 3 & 7627.2339 & -0.0003 & 0.002 \\
9 & 1 & 9 & 8 & 1 & 8 & 16536.5967 & -0.0014 & 0.002 \\
9 & 0 & 9 & 8 & 0 & 8 & 16557.3955 & -0.0009 & 0.002 \\
8 & 6 & 3 & 7 & 6 & 2 & 17239.8496 & 0.0005 & 0.002 \\
8 & 6 & 2 & 7 & 6 & 1 & 17240.0703 & 0.0003 & 0.002 \\
8 & 5 & 4 & 7 & 5 & 3 & 17306.9571 & 0.0013 & 0.002 \\
8 & 5 & 3 & 7 & 5 & 2 & 17315.4483 & 0.0017 & 0.002
\end{tabular}

Note.-Table 1 is published in its entirety in the electronic edition of the Astrophysical Journal. A portion is shown here for guidance regarding its form and content. 
TABLE 2

Spectroscopic Constants of Azulene

\begin{tabular}{|c|c|c|c|}
\hline Constant & $\begin{array}{c}\text { B3LYP/cc-pVTZ } \\
(\mathrm{MHz})\end{array}$ & $\begin{array}{c}\text { Experimental }^{\mathrm{a}} \\
(\mathrm{MHz})\end{array}$ & $\begin{array}{c}\text { Experimental }^{\mathrm{b}} \\
(\mathrm{MHz})\end{array}$ \\
\hline ...................... & 2867.9 & $2841.95182(22)$ & $2841.95225(18)$ \\
\hline$B$ & 1260.7 & $1254.843143(49)$ & $1254.843096(23)$ \\
\hline 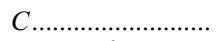 & 875.8 & $870.712741(44)$ & $870.712786(20)$ \\
\hline$\Delta_{J} \times 10^{6} \ldots \ldots \ldots$ & & $22.55(23)$ & $22.528(22)$ \\
\hline$\Delta_{J K} \times 10^{6} \ldots \ldots \ldots$ & & $37.0(14)$ & $35.45(14)$ \\
\hline$\Delta_{K} \times 10^{3} \ldots \ldots \ldots$ & & $0.1974(60)$ & $0.1893(31)$ \\
\hline$\delta_{J} \times 10^{6} \ldots \ldots \ldots \ldots$ & & $7.477(60)$ & $7.5273(80)$ \\
\hline$\delta_{K} \times 10^{6}$ & & $74.7(12)$ & $67.59(52)$ \\
\hline$\Phi_{J K} \times 10^{12} \ldots \ldots \ldots$ & & & $-23.7(25)$ \\
\hline$\Delta^{\mathrm{c}}\left(\mathrm{amu} \AA^{2}\right) \ldots \ldots$ & & -0.151 & -0.151 \\
\hline Weighted $\mathrm{rms}^{\mathrm{d}} \ldots . .$. & & 0.74 & 0.71 \\
\hline
\end{tabular}

${ }^{\text {a }}$ Centimeter-wave data only.

${ }^{b}$ Centimeter-wave and millimeter-wave data.

${ }^{\mathrm{c}}$ Inertial defect, $\Delta=I_{c}-I_{b}-I_{a}$.

d This measure is dimensionless.

(Table 3; asymmetry doublets counted once). A global fit taking all data into consideration is shown in Table 4, column (4); there is considerable improvement over the fit derived from the centimeter-wave data alone.

\subsection{Acenaphthylene}

The molecular structures of acenaphthylene and acenaphthene are very similar, differing only in the central double bond in the five-membered ring that replaces the single bond in the latter molecule (Fig. 1). Like acenaphthene, acenaphthylene is calculated at the B3LYP/cc-pVTZ level of theory to be a somewhat oblate asymmetric top $(\kappa=+0.3)$. The $b$-type dipole moment is calculated to be smaller, about one-third that of acenaphthene, or $0.31 \mathrm{D}$. Owing to the structural similarity, the theoretical rotational constants of acenaphthylene were empirically corrected for vibrationrotation interaction (Table 5), employing correction factors derived from a comparison between the theoretical and experimental rotational constants of acenaphthene (Table 4 ). This yielded improved frequency predictions, and the $6_{0,6}-5_{1,5} / 6_{1,6}-5_{0,5}$ pair of transitions was readily found at a temperature of $100^{\circ} \mathrm{C}$. For acenaphthylene, a total of 50 transitions with $3 \leq J \leq 15$ and $0 \leq K_{c} \leq 15$ in the frequency range from 7.4 to $26.7 \mathrm{GHz}$ were observed (paired transitions counted only once; see Table 6 ). The experimentally determined molecular parameters are collected in Table 5. Four out of five quartic centrifugal distortion constants $\left(\Delta_{J}, \Delta_{J K}, \Delta_{K}\right.$, and $\left.\delta_{J}\right)$ were needed to reproduce the experimental data to within the estimated uncertainties. In addition, Table 5 gives the result of an alternative fit, in which $\delta_{K}$

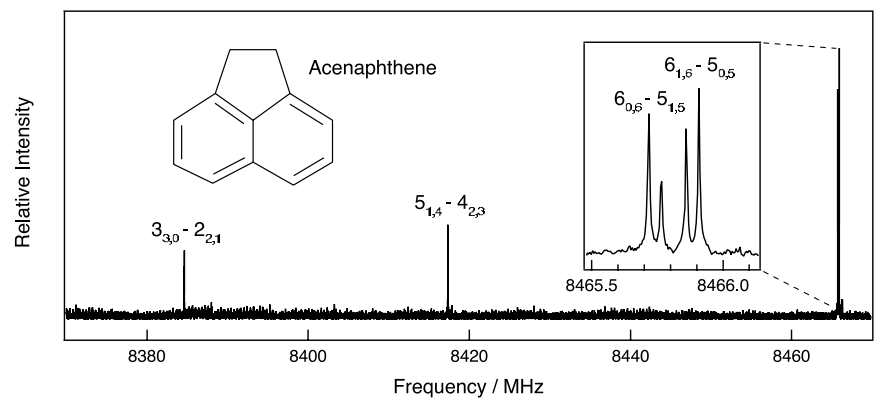

FIG. 3.-Rotational spectrum of acenaphthene at $8.4 \mathrm{GHz}$. The Doppler doubling of each line is clearly apparent in the insert on the right-hand side.
TABLE 3

Selected Experimental Rotational Transition Frequencies of ACEnaphthene

\begin{tabular}{rcccccccc}
\hline \hline & & & & & & $\begin{array}{c}\text { Frequency } \\
(\mathrm{MHz})\end{array}$ & \multicolumn{1}{c}{$\begin{array}{c}o-c \\
(\mathrm{MHz})\end{array}$} & $\begin{array}{c}\Delta \nu \\
(\mathrm{MHz})\end{array}$ \\
\hline 4 & 1 & 3 & 3 & 2 & 2 & 6994.5354 & 0.0004 & 0.002 \\
5 & 0 & 5 & 4 & 1 & 4 & 7161.2532 & 0.0007 & 0.002 \\
5 & 1 & 5 & 4 & 0 & 4 & 7162.4805 & 0.0003 & 0.002 \\
4 & 2 & 3 & 3 & 1 & 2 & 7297.9449 & -0.0027 & 0.003 \\
3 & 3 & 1 & 2 & 2 & 0 & 7788.5647 & 0.0008 & 0.002 \\
3 & 3 & 0 & 2 & 2 & 1 & 8384.6040 & 0.0022 & 0.005 \\
5 & 1 & 4 & 4 & 2 & 3 & 8417.3565 & -0.0012 & 0.002 \\
6 & 0 & 6 & 5 & 1 & 5 & 8465.7402 & -0.0002 & 0.002 \\
6 & 1 & 6 & 5 & 0 & 5 & 8465.8838 & -0.0005 & 0.002 \\
5 & 2 & 4 & 4 & 1 & 3 & 8483.6455 & -0.0022 & 0.002 \\
\hline
\end{tabular}

Note.-Table 3 is published in its entirety in the electronic edition of the Astrophysical Journal. A portion is shown here for guidance regarding its form and content.

has been adopted from acenaphthene (Table 4) and was kept fixed (see also $\S 4$ ).

\subsection{Fluorene}

Fluorene (Fig. 1, molecule 3 ) is a prolate asymmetric top molecule $\left(\kappa_{\mathrm{qcc}}=-0.86\right)$ with a calculated $b$-type dipole moment of 0.53 D. Based on predictions from the ab initio rotational constants (Table 7) a search for the $3_{3,1}-2_{2,0} / 3_{3,0}-2_{2,1}$ pair of transitions was carried out at $11.5 \mathrm{GHz}$ at a temperature of the heated nozzle of $125^{\circ} \mathrm{C}$. The lines were found to be located at 11,403 and $11,410 \mathrm{MHz}$, respectively (see Fig. 4). Shortly thereafter, another confirming pair of transitions was found, the $4_{4,1}-3_{3,0} / 4_{4,0}-3_{3,1}$ doublet at $15,759 \mathrm{MHz}$. In total, 82 lines from 8.3 to $37.5 \mathrm{GHz}$ with $2 \leq J \leq 18$ and $0 \leq K_{a} \leq 9$ were measured (Table 8; paired transitions only counted once). From a fit to these centimeter-wave data ( Table 7, col. [3]) transitions were predicted at a wavelength of $3 \mathrm{~mm}$.

A sample spectrum of fluorene showing the paired $97_{2,95}$ $96_{3,94} / 97_{3,95}-96_{2,94}$ transitions at $92.2 \mathrm{GHz}$ is shown in Figure 5. Some 20 lines of fluorene were measured at $3 \mathrm{~mm}$ and added to the centimeter-wave data to perform a global fit. In the final fit, some lines with residuals $o-c$ larger than $3 \sigma$ were omitted. The

TABLE 4

Spectroscopic Constants of Acenaphthene

\begin{tabular}{|c|c|c|c|}
\hline $\begin{array}{c}\text { Constant } \\
\text { (1) }\end{array}$ & $\begin{array}{c}\text { B3LYP/cc-pVTZ } \\
\text { (MHz) } \\
\text { (2) }\end{array}$ & $\begin{array}{c}\text { Experimental }^{\mathrm{a}} \\
(\mathrm{MHz}) \\
(3)\end{array}$ & $\begin{array}{c}\text { Experimental }^{\mathrm{b}} \\
(\mathrm{MHz}) \\
(4)\end{array}$ \\
\hline$A$ & 1416.5 & $1410.278969(61)$ & $1410.278918(26)$ \\
\hline$B$ & 1200.6 & $1193.865928(72)$ & $1193.865936(36)$ \\
\hline С & 655.0 & $652.052760(42)$ & $652.052803(17)$ \\
\hline$\Delta_{J} \times 10^{6}$. & & $30.10(28)$ & $30.01(10)$ \\
\hline$\Delta_{J K} \times 10^{6}$. & & $-48.6(10)$ & $-48.29(52)$ \\
\hline$\Delta_{K} \times 10^{6} \ldots \ldots \ldots$ & & $21.24(83)$ & $21.23(42)$ \\
\hline$\delta_{J} \times 10^{6} \ldots \ldots \ldots \ldots$ & & $-3.33(13)$ & $-3.234(46)$ \\
\hline$\delta_{K} \times 10^{6}$. & & $4.3(20)$ & $4.51(91)$ \\
\hline$\Delta^{\mathrm{c}}\left(\mathrm{amu} \AA^{2}\right) \ldots \ldots$ & & -6.608 & -6.609 \\
\hline$\mu_{b}$ (D) $\ldots \ldots \ldots \ldots$ & 0.90 & & \\
\hline Weighted $\mathrm{rms}^{\mathrm{d}} \ldots .$. & & 0.50 & 0.51 \\
\hline
\end{tabular}

a Centimeter-wave data only.

b Combined fit of centimeter- and millimeter-wave data.

c Inertial defect, $\Delta=I_{c}-I_{b}-I_{a}$.

$\mathrm{d}$ This measure is dimensionless. 
TABLE 5

Spectroscopic Constants of Acenaphthylene

\begin{tabular}{|c|c|c|c|c|}
\hline $\begin{array}{c}\text { Constant } \\
\text { (1) }\end{array}$ & $\begin{array}{c}\text { B3LYP/cc-PVTZ } \\
\text { (MHz) } \\
\text { (2) }\end{array}$ & $\begin{array}{l}\text { Scaled Constants } \\
\text { (MHz) } \\
\text { (3) }\end{array}$ & $\begin{array}{c}\text { Experimental }^{\mathrm{b}} \\
(\mathrm{MHz}) \\
\text { (4) }\end{array}$ & $\begin{array}{c}\text { Experimental } \\
(\mathrm{MHz}) \\
(5)\end{array}$ \\
\hline A & 1520.7 & 1514.0 & $1511.82609(12)$ & $1511.82605(12)$ \\
\hline B & 1228.2 & 1221.3 & $1220.63105(29)$ & $1220.63108(29)$ \\
\hline 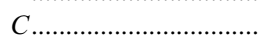 & 679.4 & 676.3 & $675.529854(65)$ & $675.529851(65)$ \\
\hline$\Delta_{J} \times 10^{6} \ldots \ldots \ldots \ldots \ldots$ & & & $31.4(26)$ & $31.4(26)$ \\
\hline$\Delta_{J K} \times 10^{6} \ldots \ldots \ldots \ldots \ldots \ldots$ & & & $-49.2(68)$ & $-50.9(68)$ \\
\hline$\Delta_{K} \times 10^{6} \ldots \ldots \ldots \ldots \ldots \ldots$ & & & $20.8(44)$ & $22.5(44)$ \\
\hline $\bar{\delta}_{J} \times 10^{6} \ldots \ldots \ldots \ldots$ & & & $-5.4(14)$ & $-5.4(14)$ \\
\hline$\delta_{K} \times 10^{6} \ldots \ldots \ldots \ldots \ldots \ldots$ & & & 0.0 & 4.5 \\
\hline$\Delta^{\mathrm{d}}\left(\mathrm{amu} \AA^{2}\right)$ & & & -0.192 & -0.192 \\
\hline$\mu_{b}$ (D) & 0.31 & & & \\
\hline 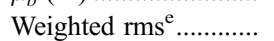 & & & 0.35 & 0.34 \\
\hline
\end{tabular}

\footnotetext{
${ }^{a}$ From acenaphthene; see text.

${ }^{\mathrm{b}} \delta_{K}$ kept fixed at zero in the analysis.

c $\delta_{K}$ constrained to value from acenaphthene (Table 4).

${ }^{\mathrm{d}}$ Inertial defect, $\Delta=I_{c}-I_{b}-I_{a}$.

e This measure is dimensionless.
}

corresponding molecular parameters are given in column (4) of Table 7. As for acenaphthene (Fig. 1, molecule 1), no sextic centrifugal distortion terms were needed, but the improvement in the quartic terms (Table 7, col. [3] vs. [4]) is clearly apparent. It should be noted that a structurally very closely related compound, carbazole $\left(\mathrm{C}_{12} \mathrm{H}_{9} \mathrm{~N}\right)$, has been investigated by FTM spectroscopy in the gas phase before (Suenram et al. 1988), where the central methylene group in fluorene is replaced by NH. As expected, the rotational constants of both molecules are similar.

\subsection{Phenanthrene}

Phenanthrene consists of three fused benzene rings (Fig. 1, molecule 5) and is the largest system investigated here theoretically (14 carbon atoms). It is calculated (B3LYP/cc-pVTZ) to be a prolate asymmetric top molecule $(A=1627.1 \mathrm{MHz}, B=$ $554.3 \mathrm{MHz}$, and $\left.C=413.424 \mathrm{MHz}, \kappa_{\mathrm{qcc}}=-0.8\right)$ with the dipole moment oriented along the main inertial $b$-axis. However, the dipole moment is calculated here to be very small, of order $0.03 \mathrm{D}$, and hence, the rotational spectrum is extremely weak. As

TABLE 6

Selected Experimental Rotational Transition Frequencies of Acenaphthylene

\begin{tabular}{rcccccccc}
\hline \hline$J^{\prime}$ & $K_{a}^{\prime}$ & $K_{c}^{\prime}$ & $J^{\prime \prime}$ & $K_{a}^{\prime \prime}$ & $K_{c}^{\prime \prime}$ & $\begin{array}{c}\text { Frequency } \\
(\mathrm{MHz})\end{array}$ & $\begin{array}{c}o-c \\
(\mathrm{MHz})\end{array}$ & $\begin{array}{c}\Delta \nu \\
(\mathrm{MHz})\end{array}$ \\
\hline 5 & 0 & 5 & 4 & 1 & 4 & 7429.4251 & -0.0004 & 0.002 \\
5 & 1 & 5 & 4 & 0 & 4 & 7432.8877 & -0.0002 & 0.002 \\
6 & 0 & 6 & 5 & 1 & 5 & 8781.6787 & 0.0000 & 0.002 \\
6 & 1 & 6 & 5 & 0 & 5 & 8782.1948 & 0.0002 & 0.002 \\
6 & 1 & 5 & 5 & 2 & 4 & 10122.8550 & -0.0001 & 0.002 \\
7 & 0 & 7 & 6 & 1 & 6 & 10132.8218 & -0.0002 & 0.002 \\
7 & 1 & 7 & 6 & 0 & 6 & 10132.8936 & -0.0007 & 0.002 \\
6 & 2 & 5 & 5 & 1 & 4 & 10153.7192 & 0.0006 & 0.002 \\
5 & 3 & 3 & 4 & 2 & 2 & 10803.7618 & 0.0023 & 0.003 \\
6 & 2 & 4 & 5 & 3 & 3 & 11248.6905 & 0.0012 & 0.002 \\
\hline
\end{tabular}

Note.-Table 6 is published in its entirety in the electronic edition of the Astrophysical Journal. A portion is shown here for guidance regarding its form and content. a consequence, phenanthrene is not a good candidate for radio astronomical searches and no attempt was made to record rotational transitions.

\section{DISCUSSION}

The PAHs azulene, acenaphthene, acenapthylene, and fluorene have been characterized by Fourier transform microwave and free-space millimeter-wave absorption spectroscopy, the latter three for the first time. The initial spectroscopic search was guided by DFT calculations at the B3LYP/cc-pVTZ level of theory and the experimental (ground-state) rotational constants are found to be very close to the theoretical (equilibrium) constants. A corresponding calculation for azulene (Table 2), for which the rotational constants had already been known prior to the present work, finds that the experimental ground-state rotational constants are somewhat smaller than the calculated equilibrium values and agree to each other within $1 \%$. As can be seen from Tables 4, 5, and 7, this

TABLE 7

Spectroscopic Constants of Fluorene

\begin{tabular}{|c|c|c|c|}
\hline $\begin{array}{l}\text { Constant } \\
\text { (1) }\end{array}$ & $\begin{array}{c}\text { B3LYP/cc-pVTZ } \\
\text { (MHz) } \\
\text { (2) }\end{array}$ & $\begin{array}{c}\text { Experimental }^{\mathrm{a}} \\
(\mathrm{MHz}) \\
(3)\end{array}$ & $\begin{array}{c}\text { Experimental }^{\mathrm{b}} \\
(\mathrm{MHz}) \\
(4)\end{array}$ \\
\hline$A$ & 2195.1 & $2176.210160(70)$ & $2176.210153(70)$ \\
\hline B & 588.2 & $586.65338(12)$ & $586.653414(70)$ \\
\hline C...... & 465.2 & $463.568993(63)$ & $463.569028(20)$ \\
\hline$\Delta_{J} \times 10^{6}$. & & $4.61(33)$ & $4.73(18)$ \\
\hline$\Delta_{J K} \times 10^{6}$ & & $-4.4(14)$ & $-4.68(84)$ \\
\hline$\Delta_{K} \times 10^{6} \ldots$ & & $98.4(14)$ & $98.4(11)$ \\
\hline 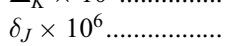 & & $1.26(16)$ & $1.243(88)$ \\
\hline$\delta_{K} \times 10^{6} \ldots \ldots \ldots \ldots \ldots \ldots \ldots \ldots \ldots \ldots \ldots$ & & $15.7(90)$ & $17.5(31)$ \\
\hline$\Delta^{\mathrm{c}}\left(\mathrm{amu} \AA^{2}\right) \ldots \ldots \ldots$ & & -3.498 & -3.498 \\
\hline$\mu_{b}$ (D) $\ldots \ldots \ldots \ldots \ldots \ldots$ & 0.53 & & \\
\hline Weighted rms ${ }^{\mathrm{d}} \ldots \ldots . . .$. & & 0.38 & 0.66 \\
\hline
\end{tabular}

a Centimeter-wave data only.

${ }^{\mathrm{b}}$ Centimeter- and millimeter-wave data.

c Inertial defect, $\Delta=I_{c}-I_{b}-I_{a}$.

$\mathrm{d}$ This measure is dimensionless. 


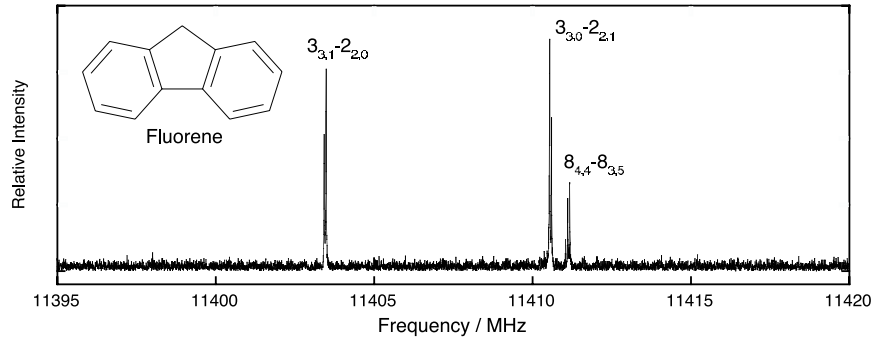

FIG. 4.- Rotational spectrum of fluorene at $11.4 \mathrm{GHz}$. In addition to the $3_{3,1}-2_{2,0} / 3_{3,0}-2_{2,1}$ pair of transitions, the $8_{4,4}-8_{3,5} Q$-branch $(\Delta J=0)$ transition is detected.

finding holds also for acenaphthene, acenaphthylene, and fluorene, respectively. An experimental value for the dipole moment had also only been obtained so far for azulene, $\mu_{a}=0.8821(24) \mathrm{D}$ (Huber et al. 2005). The corresponding B3LYP/cc-pVTZ value calculated here is $1.0 \mathrm{D}$, which is larger than the experimental value by $14 \%$. A comparison B3LYP/cc-pVTZ versus experiment for several selected carbon-chain molecules has revealed agreement to within $10 \%$ (Thorwirth et al. 2004). Hence, the values obtained here theoretically for acenaphthene $(0.90 \mathrm{D})$, acenaphthylene $(0.31 \mathrm{D})$, and fluorene $(0.53 \mathrm{D})$ are probably fairly good.

The full sets of quartic centrifugal distortion (CD) terms could be determined from the present data for acenaphthene and fluorene; for azulene, additionally, one sextic constant could be determined from the fit. For acenaphthylene, no millimeter-wave lines could be assigned with confidence. The purity of the sample purchased was $75 \%$ and as specified by the vendor (Sigma-Aldrich) some $20 \%$ of the $25 \%$ impurities were acenaphthene. While this gives a ratio of acenaphthylene/acenaphthene in the sample of $\sim 4$, the ratio of the dipole moments squared is only 0.1 . Consequently, assuming similar abundances of the two compounds in the gas phase, one expects the weak and dense millimeter-wave rotational spectrum of the "impurity" acenaphthene to be stronger by approximately a factor of 2.5 , making detection and assignment of the millimeter-wave spectrum of acenaphthylene difficult. Owing to the lack of sufficient experimental data, only four out of five quartic centrifugal distortion constants could be determined in the case of acenaphthylene. Inspection of the CD terms of acenaphthene and acenaphthylene reveals that the values are very similar, which is hardly surprising given the close structural

TABLE 8

Selected Experimental Rotational Transition Frequencies of Fluorene

\begin{tabular}{lcrrrrcrc}
\hline \hline$J^{\prime}$ & $K_{a}^{\prime}$ & $K_{c}^{\prime}$ & $J^{\prime \prime}$ & $K_{a}^{\prime \prime}$ & $K_{c}^{\prime \prime}$ & $\begin{array}{c}\text { Frequency } \\
(\mathrm{MHz})\end{array}$ & $\begin{array}{c}o-c \\
(\mathrm{MHz})\end{array}$ & $\begin{array}{c}\Delta \nu \\
(\mathrm{MHz})\end{array}$ \\
\hline 8 & 1 & 8 & 7 & 0 & 7 & 8340.8286 & -0.0013 & 0.002 \\
9 & 0 & 9 & 8 & 1 & 8 & 8469.5835 & -0.0014 & 0.002 \\
9 & 1 & 9 & 8 & 0 & 8 & 9142.3521 & -0.0001 & 0.002 \\
10 & 0 & 10 & 9 & 1 & 9 & 9499.6836 & -0.0002 & 0.002 \\
5 & 2 & 4 & 4 & 1 & 3 & 9587.2783 & -0.0016 & 0.003 \\
10 & 1 & 10 & 9 & 0 & 9 & 9970.5103 & 0.0010 & 0.002 \\
11 & 0 & 11 & 10 & 1 & 10 & 10501.2818 & -0.0001 & 0.002 \\
11 & 1 & 11 & 10 & 0 & 10 & 10823.5806 & 0.0009 & 0.002 \\
7 & 2 & 6 & 6 & 1 & 5 & 11021.2842 & -0.0021 & 0.002 \\
3 & 3 & 1 & 2 & 2 & 0 & 11403.4639 & 0.0002 & 0.002 \\
3 & 3 & 0 & 2 & 2 & 1 & 11410.5791 & 0.0004 & 0.002 \\
8 & 4 & 4 & 8 & 3 & 5 & 11411.1514 & 0.0007 & 0.002 \\
\hline
\end{tabular}

Note.-Table 8 is published in its entirety in the electronic edition of the Astrophysical Journal. A portion is shown here for guidance regarding its form and content.

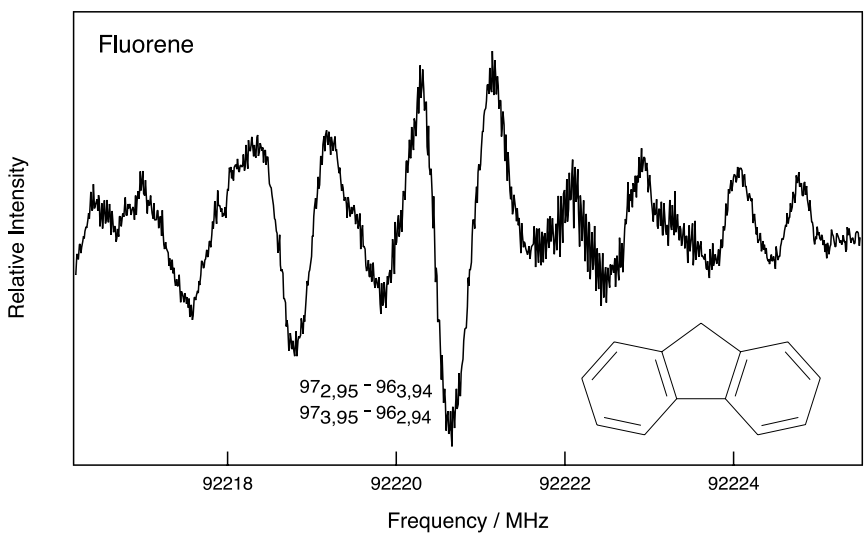

FIG. 5.- Sample spectrum of fluorene at $92 \mathrm{GHz}$. The central absorption feature is due to two collapsed rotational transitions, $97_{2,95}-96_{3,94}$ and $97_{3,95}-96_{2,94}$. The weaker transitions are thought to be vibrational satellites, i.e., pure rotational transitions from fluorene in vibrationally excited states.

similarity of the two molecules. Therefore, an alternative fit has been performed for acenaphthylene keeping $\delta_{K}$ fixed at the value previously determined for acenaphthene (Table 5, col. [5]). This approach is found to have little impact on the released distortion constants that change only within their uncertainties, but should enable a more reliable extrapolation of transition frequencies into the millimeter-wave regime.

It may be noted here briefly that spin statistics owing to the presence of pairs of equivalent hydrogen atoms are only of minor importance in the present work. For azulene with six hydrogen atoms in three equivalent pairs, the ortho: para spin-statistical weight ratio is $9: 7$, not entirely negligible in the rotational spectra. For four equivalent hydrogen pairs, as in acenaphthylene, the ortho : para ratio is 15:17 and can be taken as unity.

For a rigid planar molecule in its equilibrium configuration, the zero-point inertial defect $\Delta=I_{c}-I_{b}-I_{a}$ is zero, and for a nonplanar molecule it is negative. Because of zero-point vibration, however, inertial defects are often small and positive for many planar molecules (Gordy \& Cook 1984). There are planar molecules, however, that show negative inertial defects, because they possess energetically low-lying out-of-plane (OOP) vibrational modes (e.g., Oka 1995). These considerations apply to two of the molecules investigated here, azulene and acenaphthylene, both of which show substantial negative inertial defects of $\Delta=$ -0.15 and -0.19 amu $\AA^{2}$, respectively. For azulene, the three lowest vibrational modes are OOP modes, two of which have vibrational frequencies below $200 \mathrm{~cm}^{-1}$ (see, e.g., Kozlowski et al. [1995] for a comparison of theoretical values at different levels of theory and experimental values). The situation for acenaphthylene is found to be rather similar; a calculation at the B3LYP/6-31G* level of theory indicates that the four lowest modes are OOP vibrations. In addition to the two $b_{1}$ modes given in Banisaukas et al. (2003) at 163 and $227 \mathrm{~cm}^{-1}$, there are two vibrational modes of $\mathrm{a}_{2}$ symmetry at 209 and $367 \mathrm{~cm}^{-1}$ (B3LYP/6-31G*; scaling factor 0.97). The contribution of the OOP (methylene) hydrogen atoms to the inertial defect in case of fluorene (one pair) and acenaphthene (two pairs) manifests itself in the much more pronounced negative inertial defect values of -3.5 and $-6.6 \mathrm{amu} \AA^{2}$, respectively.

The energetically low-lying vibrational modes of fluorene and acenaphthene are also the cause of the congested spectra observed in the millimeter-wave region (e.g., Fig. 5). At room temperature many vibrational states are significantly populated, each with its own rotational spectrum. No attempt has been made here 
to assign any of these vibrational satellites to a particular vibrational mode. In contrast, as can be seen from the survey spectra in Figures 3 and 4, the microwave regions look comparably clean, which may be attributed to efficient vibrational cooling taking place during adiabatic expansion of the molecular beam.

It has been shown recently that besides pure hydrocarbon PAHs, deuterated (PADs) and nitrogen-bearing variants (PANHs) may also be present in space (Peeters et al. 2004a; Hudgins et al. 2005). The PANHs are especially interesting spectroscopically, because they should exhibit strong rotational spectra, owing to the polarity of as much as several debye, introduced by the inclusion of nitrogen in the carbon skeleton of the molecule (e.g., Mattioda et al. 2003; Hudgins et al. 2005). While the microwave spectra of simple aromatic systems incorporating nitrogen such as pyridine $\left(\mathrm{C}_{5} \mathrm{H}_{5} \mathrm{~N}\right.$; Ye et al. 2005 and references therein), pyrimidine $\left(\mathrm{C}_{4} \mathrm{H}_{4} \mathrm{~N}_{2}\right.$; Kisiel et al. 1999 and references therein), and quinoline/ isoquinoline $\left(\mathrm{C}_{9} \mathrm{H}_{7} \mathrm{~N}\right.$; Kisiel et al. 2003) have been studied by microwave/millimeter-wave spectroscopy, only very little is known about larger systems. A millimeter-wave study of phenanthridine
$\left(\mathrm{C}_{13} \mathrm{H}_{9} \mathrm{~N}\right)$, a monoaza variant of phenanthrene, has very recently been reported by McNaughton et al. (2007).

With the data here, astronomical searches for acenaphthene, ancenaphthylene, and fluorene are now possible with large radio telescopes. The transition frequencies have been determined to a few parts in $10^{7}$, corresponding to $\approx 150 \mathrm{~m} \mathrm{~s}^{-1}$ and better in equivalent radial velocity up to wavelengths of $3 \mathrm{~mm}$. Based on these data, reliable transition frequencies can be calculated from the centimeter-into the millimeter-wave regime to probe both the cold and warm molecular objects.

We thank Alfred Bauder for azulene microwave data prior to publication. This work was supported in part by NASA grant NAG 05-9379 and NSF grant CHE-0353693. S. Thorwirth is grateful to the Alexander von Humboldt-Foundation for a Feodor Lynen research fellowship awarded for the period from 2003 April to 2005 April. P. Theule would like to thank the Swiss National Science Foundation for a research fellowship.
Allamandola, L. J., Tielens, A. G. G. M., \& Barker, J. R. 1985, ApJ, 290, L25

Banisaukas, J., et al. 2003, J. Phys. Chem. A, 107, 782

Becke, A. D. 1993, J. Chem. Phys., 98, 5648

Dunning, T. H. 1989, J. Chem. Phys., 90, 1007

Frisch, M. J., et al. 2003, Gaussian 03, Revision B.04 (Wallingford: Gaussian, Inc.)

Gordy, W., \& Cook, R. L. 1984, Microwave Molecular Spectra (3rd ed.; New York: Wiley)

Gottlieb, C. A., Myers, P. C., \& Thaddeus, P. 2003, ApJ, 588, 655

Huber, S., Grassi, G., \& Bauder, A. 2005, Mol. Phys., 103, 1395

Hudgins, D. M., Bauschlicher, C. W., \& Allamandola, L. J. 2005, ApJ, 632, 316

Kisiel, Z., Desyatnik, O., Pszczolkowski, L., Charnley, S. B., \& Ehrenfreund, P. 2003, J. Mol. Spectrosc., 217, 115

Kisiel, Z., Pszczolkowski, L., Lopez, J. C., Alonso, J. L., Maris, A., \& Caminati, W. 1999, J. Mol. Spectrosc., 195, 332

Kozlowski, P. M., Rauhut, G., \& Pulay, P. 1995, J. Chem. Phys., 103, 5650

Leger, A., \& Puget, J. L. 1984, A\&A, 137, L5

Lovas, F. J., McMahon, R. J., Grabow, J.-U., Schnell, M., Mack, J., Scott, L. T., \& Kuczkowski, R. L. 2005, J. Am. Chem. Soc., 127, 4345

Mattioda, A. L., Hudgins, D. M., Bauschlicher, C. W., Rosi, M., \& Allamandola, L. J. 2003, J. Phys. Chem. A, 107, 1486

McCarthy, M. C., Chen, W., Travers, M. J., \& Thaddeus, P. 2000, ApJS, 129, 611

McCarthy, M. C., Travers, M. J., Kovacs, A., Gottlieb, C. A., \& Thaddeus, P. 1997, ApJS, 113, 105

\section{EFERENCES}

McNaughton, D., Godfrey, P. D., Brown, R. D., \& Thorwirth, S. 2007, Phys. Chem. Chem. Phys., 9, 591

Oka, T. 1995, J. Mol. Struct., 352/353, 225

Oomens, J., Tielens, A. G. G. M., Sartakov, B. G., von Helden, G., \& Meijer, G. 2003, ApJ, 591, 968

Peeters, E., Allamandola, L. J., Bauschlicher, C. W., Hudgins, D. M., Sandford, S. A., \& Tielens, A. G. G. M. 2004a, ApJ, 604, 252

Peeters, E., Allamandola, L. J., Hudgins, D. M., Hony, S., \& Tielens, A. G. G. M. 2004b, in ASP Conf. Ser. 309, Astrophysics of Dust, ed. A. N. Witt, G. C. Clayton, \& B. T. Draine (San Francisco: ASP), 141

Pickett, H. M. 1991, J. Mol. Spectrosc., 148, 371

Salama, F. 1999, in Solid Interstellar Matter: The ISO Revolution, ed. L. d'Hendecourt, C. Joblin, \& A. Jones (Berlin: Springer), 65

Suenram, R. D., Lovas, F. J., Fraser, G. T., \& Marfey, P. S. 1988, J. Mol. Struct., 190, 135

Thorwirth, S., McCarthy, M. C., Dudek, J. B., \& Thaddeus, P. 2004, J. Mol. Spectrosc., 225, 93

Thorwirth, S., McCarthy, M. C., Gottlieb, C. A., Thaddeus, P., Gupta, H., \& Stanton, J. F. 2005, J. Chem. Phys., 123, 054326

Tielens, A. G. G. M., \& Peeters, E. 2004, in The Dense Interstellar Medium in Galaxies, ed. S. Pfalzner et al. (Berlin: Springer), 497

Tobler, H. J., Bauder, A., \& Günthard, H. H. 1965, J. Mol. Spectrosc., 18, 239

Ye, E., Bettens, R. P. A., De Lucia, F. C., Petkie, D. T., \& Albert, S. 2005, J. Mol. Spectrosc., 232, 61 